\title{
Sciatic nerve stiffness is not changed immediately after a slump neurodynamics technique
}

\author{
Tiago Neto ${ }^{1,2}$ \\ Sandro R Freitas ${ }^{1,3}$ \\ Ricardo J Andrade ${ }^{1,4}$ \\ João Gomes ${ }^{1}$ \\ João Vaz ${ }^{1,3}$ \\ Bruno Mendes ${ }^{1,3}$ \\ Telmo Firmino ${ }^{1,3}$ \\ Antoine Nordez ${ }^{4}$ \\ Raúl Oliveira ${ }^{1}$ \\ 1 Faculdade de Motricidade Humana, Universidade \\ de Lisboa, Lisbon, Portugal \\ 2 Lunex University, Faculty of Health Sciences, \\ Differdange, Luxembourg \\ 3 Benfica LAB, Sport Lisboa e Benfica, Lisboa, Portugal \\ 4 University of Nantes, Laboratory "Movement, \\ Interactions, Performance" (EA 4334), Faculty \\ of Sport Sciences, Nantes, France
}

Corresponding author:

Sandro R Freitas

Faculdade de Motricidade Humana,

Estrada da Costa 1499-002

Cruz Quebrada - Dafundo, Lisboa, Portugal

E-mail:sfreitas@fmh.ulisboa.pt

\section{Summary}

Background: Neurodynamics techniques aim to assess and improve neural mechanosensitivity. However, there is no in vivo evidence regarding the mechanical effects of these techniques in the nerve stiffness. This study examined the immediate effects of a slump neurodynamics technique in the sciatic nerve shear wave velocity (SWV. i.e. an index of stiffness) using ultrasound-based elastography.

Methods: Fourteen healthy participants were included in this experiment. Sciatic SWV and ankle passive torque were measured during a passive ankle dorsiflexion motion $(2 \% \mathrm{~s})$, before and immediately after 3 minutes of slump neurodynamics technique, randomly applied to one lower limb. The contralateral limb served as control.

Results: The slump intervention did not change the sciatic SWV $(P=0.78)$, nor the dorsiflexion passive torque $(P=0.14)$, throughout the ankle dorsiflexion motion. Excellent values of intra-rater repeatability
(ICC=0.88, 0.68-0.96), and low values of standard error of measurement $(0.59 \mathrm{~m} / \mathrm{s}, 0.35-1.15 \mathrm{~m} / \mathrm{s})$, were observed for the SWV measurements.

Conclusions: The sciatic nerve stiffness of healthy participants did not change immediately after a slump neurodynamics technique, suggesting a compliance of the neural tissue to tensile loads. However, these results ought to be confirmed using other neurodynamics techniques and in other populations (e.g. peripheral neuropathies).

Level of evidence: III.

KEY WORDS: elastography, nerve biomechanics, shear wave velocity.

\section{Introduction}

Neurodynamics techniques, such as the slump position, are frequently used by health professionals to assess and improve neural mechanosensitivity ${ }^{1}$. Its use is based on the assumption of improving the mechanical properties of nerves and their relationship with mechanical interfaces ${ }^{2}$. However, to our knowledge, such benefits in humans have never been confirmed, in vivo.

The effects of neurodynamics techniques on the peripheral nerves have been reported by several ex vi$v o^{3,4}$ and in vivo studies ${ }^{5,6}$. Investigations using animal models have shown the viscoelastic behaviour of peripheral nerves, showing for instance a $30 \%$ reduction in stress relaxation after 5 min of constant strain (i.e. $6 \%{ }^{7}$. Using human cadavers, a recent study reported strain values of approximately $8 \%$ for both the sciatic and tibial nerve, during straight leg raise manoeuvres ${ }^{3}$. Despite providing valuable information, these studies were not conducted in vivo, therefore conclusions are limited regarding the effects of neurodynamics in human nerves. In the recent years, B-mode ultrasonography has been used to assess in real time, the mechanical properties of human peripheral nerves ${ }^{8,9}$. Transverse, and mainly longitudinal neural excursion have been extensively examined, with this technology, in the median $^{10}$, tibial ${ }^{11}$, or in the sciatic nerve ${ }^{9}$, following different neurodynamics techniques. However, B-mode ultrasonography fails to provide information about the forces acting upon a nerve, such as stiffness. Peripheral nerves, as viscoelastic structures, have the ability to elongate when submitted to a certain load, which is the definition of stiffness ${ }^{12}$. 
Nowadays, it is possible to infer about the stiffness of soft tissues due to shear wave elastography. This method enables a quantitative measure of tissue shear wave velocity, which are the product of tissue displacement following ultrasound push beams ${ }^{13}$. The velocity of shear waves is correlated with the stiffness of tissues $^{13}$. This technique has very recently been used to reliably measure the stiffness of the median ${ }^{14}$, tibial ${ }^{15}$, and sciatic nerves ${ }^{16}$, in both passive and active conditions. Moreover, shear wave elastography was also used to determine inter-limb differences in the stiffness of peripheral nerves, in people with chronic neuropathies ${ }^{14,17}$. However, it is still to be determined if, and how, neural stiffness is changed by performing neurodynamics techniques.

This study aimed to determine, in vivo, the immediate effects of a neurodynamics technique applied in a slump position in the sciatic stiffness, using shear wave elastography. We hypothesized that the slump neurodynamics technique would induce an immediate decrease of the sciatic nerve stiffness of healthy participants.

\section{Materials and methods}

\section{Participants}

Nineteen healthy volunteers were invited to participate in this study. Participants reported no neuromuscular disorders to the lower limbs or lower back, and signed the informed consent accordingly to the Declaration of Helsinki. This study was approved by the local Ethics Council (CEFMH Approval number: $3 / 2015)$, and follows the ethical standards of this Journal, as described by Padulo et al. ${ }^{18}$.

\section{Equipment}

Dynamometry

An isokinetic dynamometer (Biodex system 3 research, Shirley, NY, USA) was used to passively dorsiflex the ankle joint $(2 \%$ s), while ankle angle and torque were sampled at $1000 \mathrm{~Hz}$. Participants laid prone with the lateral malleolus aligned to the dynamometer axis (Fig. $1 \mathrm{~A}$ ). The neutral position of the ankle $\left(0^{\circ}\right)$ was defined by the perpendicular position between the foot and leg, and determined by using an inclinometer.

\section{Shear wave elastography}

An ultrasound scanner (Aixplorer, version 10.0; Supersonic Imagine, Aix-en-Provence, France) was used to assess $(1 \mathrm{~Hz})$ the sciatic shear wave velocity with a linear transducer array (SL 10-2 MHz, Super Linear, Vermon, Tours, France) in the muscularskeletal preset (penetrate mode, smoothing level 9/9, and the persistence off). The maximal shear wave velocity scale value was set as $17.0 \mathrm{~m} / \mathrm{s}$. The sciatic nerve was first identified transversely (Fig. 1 B) by scanning the posterior thigh in B-mode, approximately $10 \mathrm{~cm}$ below the gluteal fold (Fig. $1 \mathrm{~A}$ ), which was described as the best region for the sciatic nerve ultrasonographic assessments ${ }^{19}$. Then, the probe was orientated longitudinally until both superficial and deep epineurium could be observed (Fig. 1 C). The probe location was marked in the skin with a waterproof marker, so repeated measures could be performed always in the same location. Clip videos with both B-mode and shear wave elastography modes displayed were recorded during measurements.

\section{Electromyography}

Surface electromyography (EMG) was used to record muscle activation, and to ensure a passive nature to the ankle motion. A telemetric system (Plux, Lisbon, Portugal) was used, and surface electrodes (Ambu $R$ BlueSensor N, Copenhagen, Denmark) were placed in the semitendinosus (ST), medial gastrocnemius (MG), and tibialis anterior (TA) mid-belly of both lower limbs, through guidance of ultrasound (B-mode). Signals were sampled at $1 \mathrm{kHz}$ rate, amplified ( $x$ 1000) and digitized (8 $-500 \mathrm{~Hz}$ bandwidth) before analysis. Resting EMG activity was deducted from the EMG values collected during the ankle dorsiflexion passive motion. EMG values were later normalized to the maximal isometric voluntary contraction (MIVC).

\section{Procedures}

Participants visited the laboratory in one single session. Before testing, the experimental lower limb (i.e. the limb subjected to the slump intervention) and the control (i.e. contralateral) lower limb were randomly defined. Pre and post tests were performed with the participants laid prone in the dynamometer and the pelvis firmly strapped. The participants' maximal passive ankle dorsiflexion range of motion (ROM) was determined by using a hand-held stop button. The ankle movement was performed at $2 \%$, and the participants were instructed to stop the movement when they reached the point of stretching discomfort. Approximately 3 min after the ROM determination, four plantarflexion-dorsiflexion cycles, starting from $40^{\circ}$ of plantarflexion to the maximal dorsiflexion angle, were performed at $5 \%$ sor conditioning purposes ${ }^{20}$. Thereafter, the shear wave velocity of the sciatic nerve and the ankle torque-angle were assessed in two maximal dorsiflexion ROM repetitions $(2 \%$ s). Between the two repetitions ( $1 \mathrm{~min}$ rest), the probe was removed from the site of measurement, for repeatability purposes. After a 2 min rest period, the slump test sequence was performed to the experimental limb (Fig. 2). Briefly, participants were seated with their hands behind the back, and were asked to flex their lumbar and thoracic spine into a slump position. Afterwards, cervical spine flexion was added, and passively maintained in this position by an examiner. While the control limb stayed relaxed, the knee was passively extended by a second examiner, while the ankle was maximally dorsiflexed, until the point of stretching discomfort. This position was statically maintained during $3 \mathrm{~min}$ (Fig. 2). Immediately after the slump protocol, participants were re-positioned in prone position for the post testing. The post measurements were always performed first in the experimental limb, in order to rapidly assess the effects of the slump intervention. Finally, 3 MIVC repetitions for the knee flexors, plantar and dor- 

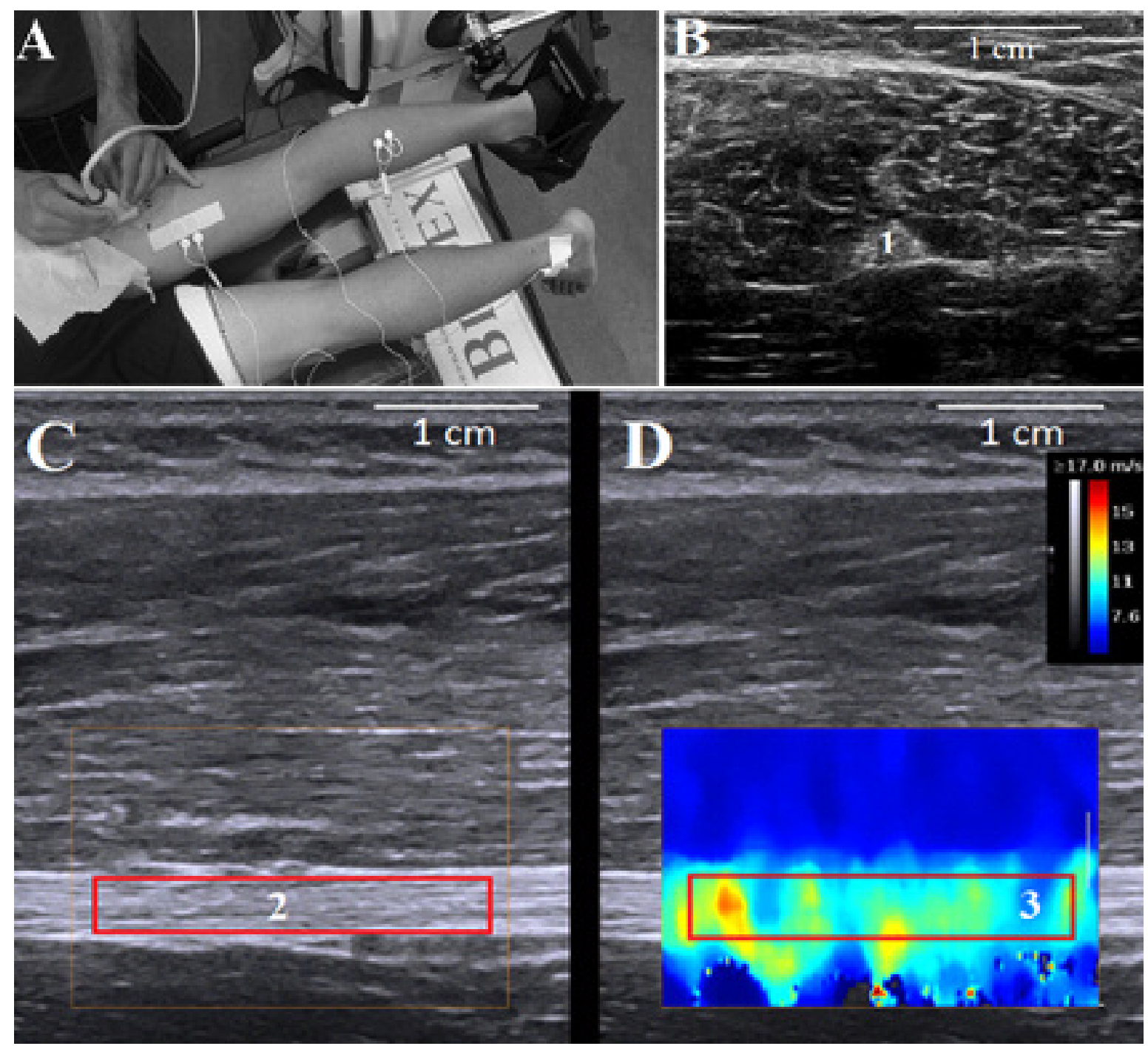

Figure 1 A-D. (A) Testing set-up. B-mode sonograms of sciatic nerve in (B) cross-sectional and (C) longitudinal views. The (D) elastogram window was defined over the nerve section, and the largest area within the epineurium was considered as region of interest. Legend: 1, Sciatic nerve cross-sectional area; 2, Sciatic nerve longitudinal view; 3 , Sciatic nerve elastogram region of interest.

siflexors ( $1 \mathrm{~min}$ rest between repetitions) were performed for EMG normalization purposes.

\section{Data processing}

All data acquisition was synchronized by using an external trigger, saved in a personal computer, and processed using customized MatLab routines (The Mathworks, Natick, USA). Briefly, in the sonograms clips, the sciatic region of interest was determined by selecting the largest area within the epineurium in the elastogram window (Fig. 1 D). This procedure was repeated for each frame (i.e. every second), to ensure that the region of interest would not be affected by the nerve motion during the maneuvers. When selecting the region of interest, care was taken to avoid regions of saturation (i.e. values higher than the ones supported by the ultrasound device), since they are considered as artifacts. Shear wave velocity values were calculated by converting the color pixels (in accordance with the scale) and averaged for statistical analysis. The sonogram clips with incomplete elastogram (i.e. elastogram in which the nerve region showed a partial absence of shear wave signal) were excluded from analysis.

As the ankle maximal ROM was different between the participants, the ankle angles were normalized to the maximal ROM. Ankle range until $80 \%$ of maximal ROM was considered for analysis. This ROM cut-off was also used in a previous study ${ }^{16}$, since the elastogram in some participants reached the maximal value of the scale (i.e. $17 \mathrm{~m} / \mathrm{s}$ ), and considerable artefacts occur above this ankle ROM.

\section{Statistical analysis}

The intraclass coefficient correlation (ICC 2, 1) was determined to assess the intra-rater repeatability of the shear wave velocity measurements. In addition, 


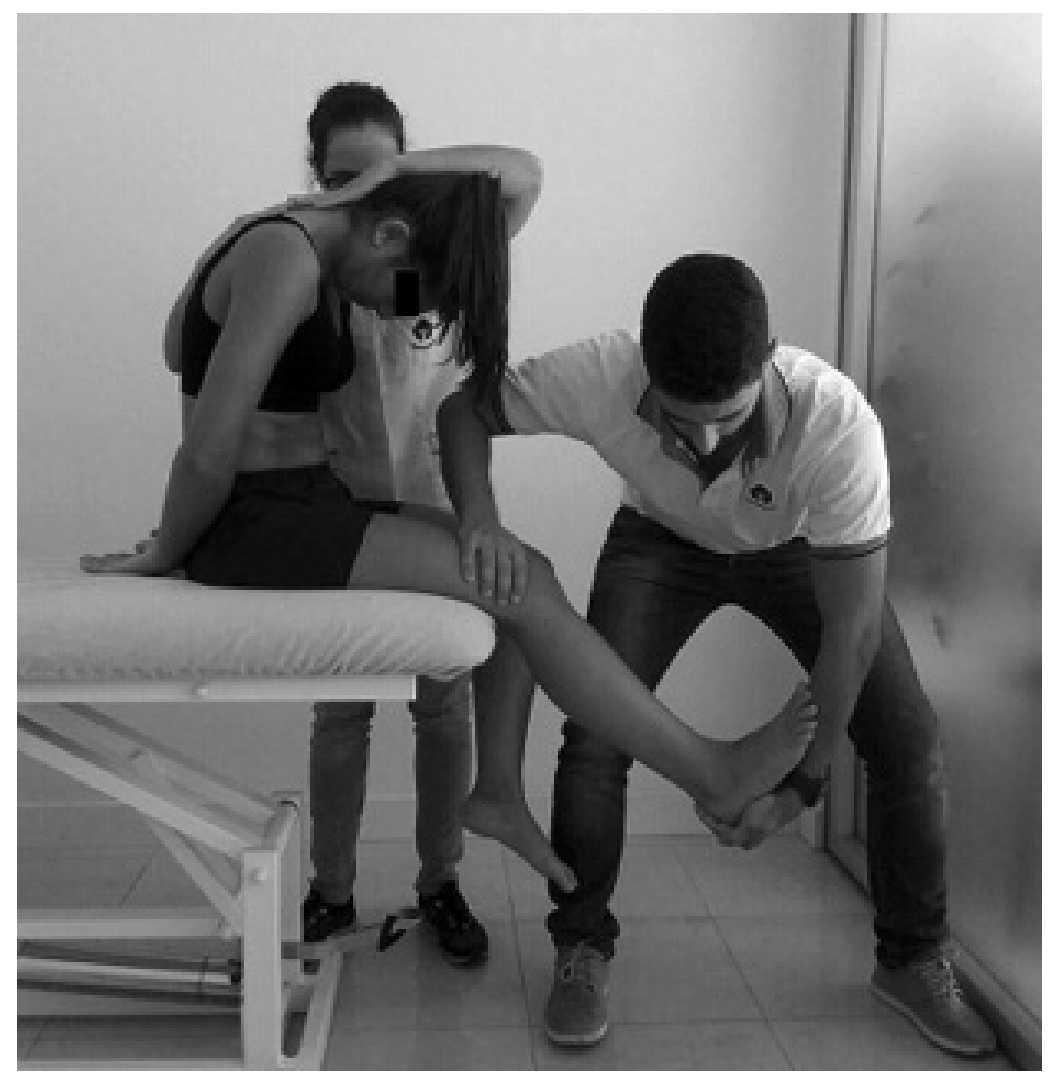

Figure 2. Slump test position.

the standard error of measurement (SEM), the minimal detectable difference (MDD), and the coefficient of variation (CV) were determined. The SEM was calculated as follows: $S E M=\sqrt{ }$ MSE, where MSE represents the square root of the error mean square, obtained from the 2-way random effects analysis of variance. The MDD was determined by the formula MDD=SEM $\times 1.96 \times \sqrt{ } 2$, and the $C V$ was calculated by dividing the standard deviation over the mean ${ }^{21}$. The repeatability parameters were determined for every $10 \%$ interval of the ankle ROM.

The normality of the data was confirmed using the Shapiro-Wilk test. A 3-way repeated measures ANOVA [limb (control, experimental) $\times$ angle $(0,10,20$, $30,40,50,60,70$, and $80 \%$ of maximal ROM) $\times$ condition (pre, post)] was calculated to determine the effects of the intervention in the shear wave velocity of the sciatic nerve and in the ankle passive torque. The Bonferroni correction method was used for post-hoc analysis. Statistical significance was set at 0.05 .

\section{Results}

During data collection, 5 participants $(3$ males, 2 females; $30.4 \pm 10.1$ years; $75.6 \pm 14.3 \mathrm{~kg} ; 1.75 \pm 0.1$ $\mathrm{m})$ were excluded due to an incomplete shear wave elastogram. Consequently, the data analysis was performed in 14 participants (11 males, 3 females; 28.4 \pm 6.7 years; $69.6 \pm 8.7 \mathrm{~kg} ; 1.74 \pm 0.08 \mathrm{~m}$ ).
During all the tests, a $1.5 \% \pm 1.1 \%, 2.0 \% \pm 0.9 \%$, and $2.8 \% \pm 1.3 \%$ of muscle activation were observed for the semitendinosus, medial gastrocnemius, and tibialis anterior muscles, respectively.

The intra-rater repeatability outcomes for sciatic nerve shear wave velocity assessment are presented in Table I.

In respect to the sciatic shear wave velocity (Fig. 3 A), no effects were found for the $\operatorname{limb}(F 1,13=0.648$, $p=0.435$ ) or condition ( $F 1,13=0.082, p=0.779)$ variables. However, a significant effect was found for the angle variable $(F 8,104=47.604, p<0.001)$. Post hoc analysis revealed an increased shear wave velocity at 50 to $80 \%$ of ankle ROM compared to the $0 \%$ of ankle ROM in both the experimental $(p=0.04)$ and control $(p=0.01)$ limbs, and in both the pre $(p=0.01)$ and post $(p=0.02)$ intervention. Similarly, no main effects were observed for the $\operatorname{limb}(F 1,13=0.342$, $\mathrm{p}=0.569)$ and condition $(\mathrm{F} 1,13=2.498, \mathrm{p}=0.138)$ variables regarding the ankle passive torque (Fig. $3 \mathrm{~B}$ ). However, a main effect was observed for the angle variable $(F 8,104=51.901, p<0.001)$. In both conditions, ankle passive torque significantly increased across all tested ROM $(p<0.01)$.

\section{Discussion}

This study used shear wave elastography to test the hypothesis that a slump neurodynamics technique 
Table I. Intra-rater repeatability values of the sciatic nerve shear wave velocity, for every $10 \%$ interval of the total ankle range of motion. ICC, Intraclass correlation coefficient; SEM, Standard error of measurement; MDD, Minimal detectable difference; CV, Coefficient of variation.

\begin{tabular}{lllll}
\hline$\%$ of ankle ROM & ICC $(95 \% \mathbf{C l})$ & SEM $(\mathrm{m} / \mathbf{s})$ & CV $(\%)$ & MDD $(\mathrm{m} / \mathbf{s})$ \\
\hline $\mathbf{0}$ & $0.93[0.79-0.98]$ & 0.46 & 4.31 & 1.27 \\
$\mathbf{1 0}$ & $0.93[0.81-0.98]$ & 0.42 & 4.78 & 1.18 \\
$\mathbf{2 0}$ & $0.88[0.67-0.96]$ & 0.53 & 5.42 & 1.48 \\
$\mathbf{3 0}$ & $0.89[0.69-0.96]$ & 0.51 & 4.97 & 1.43 \\
$\mathbf{4 0}$ & $0.95[0.84-0.98]$ & 0.35 & 3.38 & 0.98 \\
$\mathbf{5 0}$ & $0.89[0.70-0.96]$ & 0.54 & 5.46 & 1.51 \\
$\mathbf{6 0}$ & $0.88[0.68-0.96]$ & 0.57 & 5.75 & 1.58 \\
$\mathbf{7 0}$ & $0.83[0.54-0.94]$ & 0.80 & 6.72 & 2.22 \\
$\mathbf{8 0}$ & $0.74[0.38-0.91]$ & 1.15 & 8.66 & 3.19 \\
\hline
\end{tabular}

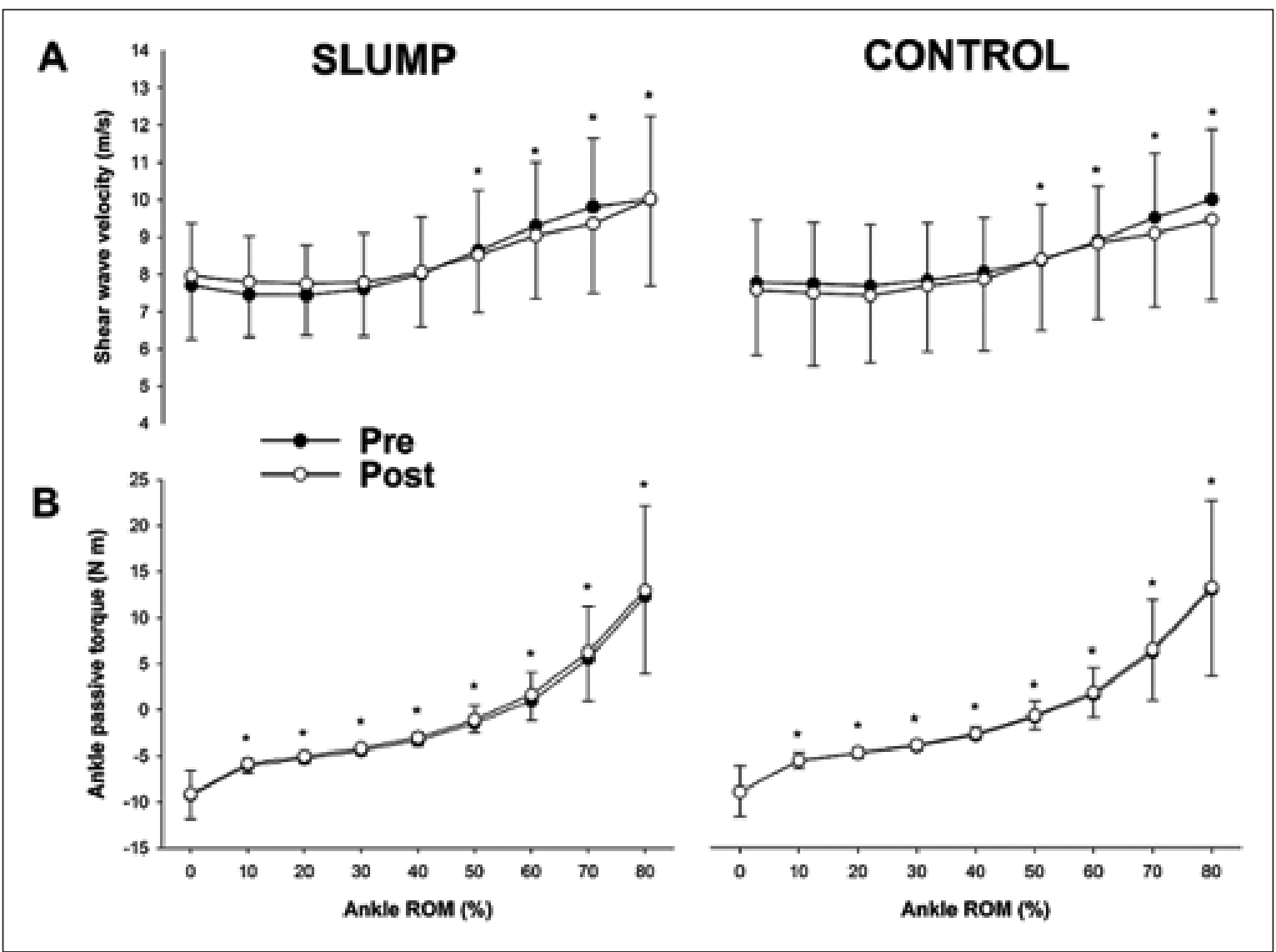

Figure $3 \mathrm{~A}$, B. (A) Shear wave velocity of the sciatic nerve and (B) ankle passive torque during the ankle passive rotation from $40^{\circ}$ of plantar flexion ( $0 \%$ ) to $80 \%$ of maximal dorsiflexion range of motion (ROM) before (pre) and after (post) the slump and control interventions. ${ }^{*}$ Statistical different from the initial ankle position ( $0 \%$ of ROM).

would induce an immediate decrease of the sciatic nerve stiffness in healthy participants. The findings from this investigation led us to reject the hypothesis previously stated. Our hypothesis was based on the immediate effects of this technique in healthy peo$\mathrm{ple}^{22}$, and also on the knowledge of neural biomechanics, where it has been shown that peripheral nerves present stress relaxation when subjected to a 
constant strain ${ }^{3,7}$. However, we must keep in mind that most of this knowledge comes from animal or cadaveric studies, which use elongation intensities close to, or even beyond, the elastic limit of the neural tissue (i.e. the point where the nerve can resume its original shape ${ }^{23}$, and for periods superior to the 3 minutes used in this study. Moreover, live human peripheral nerves, in situ, have a different mechanical behaviour (e.g. different stress-strain curves), when compared to nerves excised ${ }^{23}$, proving the influence of the surrounding tissues in neural mechanical properties. Considering all these aspects, comparisons between these results and the ones reported for animal/cadaveric investigations must be made with caution. Nevertheless, we can say that this particular neurodynamics technique, applied in healthy participants, did not influence the sciatic nerve stiffness.

Another possible explanation for the lack of significant differences may rely on the site of measurement for the sciatic nerve stiffness. In this study we measured the sciatic nerve shear wave velocity in the posterior thigh (i.e. below the gluteal fold), a site described as a location with good ultrasonic visibility and where the nerve is more superficial ${ }^{19}$. However, it has been shown that the sciatic nerve roots exhibit large displacement during bilateral SLR manoeuvres $^{24}$, possibly implying large strain in the nerve roots. Although, to our knowledge, no studies have ever measured neural stiffness at the nerve roots level (i.e. which may be difficult to achieve with ultrasonography considering the wave reflection by the bone structures surrounding the nerve root $)^{25}$, we hypothesize that measurements closer to the nerve roots of the sciatic might yield larger differences in stiffness, compared to more distal locations, and following neurodynamics interventions.

The results from this study showed that the stiffness of the sciatic nerve becomes significantly higher as the ankle is rotated towards dorsiflexion. Specifically, significant changes in shear wave velocity were seen after $50 \%$ of ankle ROM. This corresponds, in average, to $2^{\circ}$ of plantarflexion, which means that this may be the point where the sciatic nerve starts to build up more tension during ankle dorsiflexion with the knee fully extended. This is consistent with the findings of Andrade et al. ${ }^{16}$, who used an experimental set-up similar to ours, and showed significant increases in the sciatic stiffness during ankle dorsiflexion, with the knee in full extension. In another study with similar results ${ }^{15}$, it was observed a $136 \%$ increase in the tibial nerve shear wave velocity, when changed from a slack length position (i.e. hip and foot in neutral position, knee flexed at $90^{\circ}$ ) to a neural tension position (i.e. hip flexed, with the knee fully extended and the ankle dorsiflexed).

In regards to the repeatability of the shear wave velocity measurements, we observed good intra-rater repeatability ( $\mathrm{ICC}=0.74-0.95$; $\mathrm{CV}=3.4-8.9 \%$ ) for measuring the sciatic nerve stiffness during passive ankle dorsiflexion up to $80 \%$ of maximal ROM. Our results are consistent with a previous study ${ }^{16}$ conducted also in the sciatic nerve during ankle dorsiflexion, which showed similar CV values $(<8.0 \%)$ and slightly higher ICC values $(0.92-0.98)$. Not surprisingly, we observed a tendency for a lower repeatability as the ankle dorsiflexion ROM increased. This is probably due to the building up of tension in all tissues surrounding the nerve (i.e. muscle and connective tissue), and also the nerve itself. The increase in tension may cause the transverse and superficial movements previously observed in the tibial and sciatic nerves during, respectively, ankle dorsiflexion and knee extension manoeuvres ${ }^{9,26}$, affecting the repeatability of the shear wave velocity measurement. Moreover, the increasingly higher stiffness levels of the sciatic nerve were observed in the elastogram as saturation points (i.e. values of shear wave velocity above the limit of the scale used). Considering this situation, we had to exclude 5 participants due to unfilled shear wave elastogram windows. We have noted that these participants had greater thickness of both the subcutaneous adipose tissue and the deep fascia. Altogether, this may act as a barrier for the supersonic push to travel through the deepest tissues, such as the sciatic nerve (i.e. in average located at a depth of 4-5 cm). Future studies using shear wave elastography should consider these limitations when assessing peripheral nerves, especially deep nerves such as the sciatic.

In addition to the limitations previously mentioned, we acknowledge that the $3 \mathrm{~min}$ duration of the intervention is not representative of a clinical practice context. Studies using neurodynamics techniques in a slump position do not use durations longer than $30 s^{27,28}$. However, we wanted to ensure that the neural tissue had time enough to adjust to the tensile load, and eventually to exhibit stress relaxation. Moreover, we did not measure the stiffness of the surrounding tissues, such as the long head of the biceps femoris. It should be relevant, in future research, to see if: 1) there is a relation between the stiffness of the sciatic nerve and the stiffness of the surrounding tissues; and 2) if the stiffness of the sciatic nerve is influenced by the stiffness of the surrounding tissues.

\section{Conclusions}

This study showed that a slump neurodynamics technique did not result in a decrease of the sciatic nerve stiffness, in healthy participants. These findings suggest that neurodynamics interventions which aim at loading the sciatic nerve, in healthy people, do not alter the mechanical properties of the neural tissue. Weather these findings are also observed in other techniques (e.g. straight leg raise), or in other populations (e.g. people with sciatica), is yet to be determined.

\section{Conflict of interest}

None to declare. 


\section{References}

1. Maitland GD. Negative disc exploration: positive canal signs. Aust J Physiother. 1979 Jul;25(3):129-34.

2. Butler DS. The Sensitive Nervous System. Noigroup Publications. 2000:431.

3. Boyd BS, Topp KS, Coppieters MW. Impact of movement sequencing on sciatic and tibial nerve strain and excursion during the straight leg raise test in embalmed cadavers. J Orthop Sports Phys Ther. 2013 Jun;43(6):398-403.

4. Phillips JB, Smit X, De Zoysa N, Afoke A, Brown RA. Peripheral nerves in the rat exhibit localized heterogeneity of tensile properties during limb movement. J Physiol. 2004 Jun 15;557(Pt 3):879-887.

5. Ellis RF, Hing WA, McNair PJ. Comparison of longitudinal sciatic nerve movement with different mobilization exercises: an in vivo study utilizing ultrasound imaging. J Orthop Sports Phys Ther. 2012 Aug;42(8):667-675.

6. Ridehalgh C, Moore A, Hough A. Repeatability of measuring sciatic nerve excursion during a modified passive straight leg raise test with ultrasound imaging. Man Ther. 2012;17(6):572576.

7. Kwan MK, Wall EJ, Massie J, Garfin SR. Strain, stress and stretch of peripheral nerve. Rabbit experiments in vitro and in vivo. Acta Orthop Scand. 1992 Jun;63(3):267-72.

8. Coppieters MW, Butler DS. Do "sliders" slide and "tensioners" tension? An analysis of neurodynamic techniques and considerations regarding their application. Man Ther. 2008 Jun;13 (3):213-221.

9. Ridehalgh C, Moore A, Hough A. Sciatic nerve excursion during a modified passive straight leg raise test in asymptomatic participants and participants with spinally referred leg pain. Man Ther. 2015 Aug;20(4):564-569.

10. Hough AD, Moore AP, Jones MP. Reduced longitudinal excursion of the median nerve in carpal tunnel syndrome. Arch Phys Med Rehabil. 2007 May;88(5):569-576.

11. Carroll M, Yau J, Rome K, Hing W. Measurement of tibial nerve excursion during ankle joint dorsiflexion in a weightbearing position with ultrasound imaging. J Foot Ankle Res. 2012 Mar 8:5(1):5.

12. Haftek J. Stretch injury of peripheral nerve. Acute effects of stretching on rabbit nerve. J Bone Joint Surg Br. 1970 May 52(2):354-365.

13. Bercoff J, Tanter M, Fink M. Supersonic shear imaging: a new technique for soft tissue elasticity mapping. IEEE Trans Ultrason Ferroelectr Freq Control. 2004 Apr;51(4):396-409.

14. Kantarci F, Ustabasioglu FE, Delil S, Olgun DC, Korkmazer B, Dikici AS, et al. Median nerve stiffness measurement by shear wave elastography: a potential sonographic method in the diagnosis of carpal tunnel syndrome. Eur Radiol. 2014 Feb; 24(2):434-440.

15. Greening J, Dilley A. Posture-induced changes in peripheral nerve stiffness measured by ultrasound shear-wave elastography. Muscle Nerve. $2017 \mathrm{Feb} ; 55(2): 213-222$.

16. Andrade RJ, Nordez A, Hug F, Ates F, Coppieters MW, Pezarat-Correia $\mathrm{P}$, et al. Non-invasive assessment of sciatic nerve stiffness during human ankle motion using ultrasound shear wave elastography. J Biomech. 2016 Feb 8;49(3):326331.

17. Dikici AS, Ustabasioglu FE, Delil S, Nalbantoglu M, Korkmaz B, Bakan S, et al. Evaluation of the Tibial Nerve with ShearWave Elastography: A Potential Sonographic Method for the Diagnosis of Diabetic Peripheral Neuropathy. Radiology. 2017 Feb;282(2):494-501.

18. Padulo J, Oliva F, Frizziero A, Maffulli N. Muscles, Ligaments and Tendons Journal - Basic principles and recommendations in clinical and field science research: 2016 update. MLTJ. 2016;6(1):1-5.

19. Bruhn J, Van Geffen G-J, Gielen MJ, Scheffer GJ. Visualization of the course of the sciatic nerve in adult volunteers by ultrasonography. Acta Anaesthesiol Scand. 2008 Oct;52(9): 1298-1302.

20. Nordez A, McNair P, Casari P, Cornu C. Acute changes in hamstrings musculo-articular dissipative properties induced by cyclic and static stretching. Int J Sports Med. 2008 May; 29(5):414-418

21. Weir JP. Quantifying test-retest reliability using the intraclass correlation coefficient and the SEM. J Strength Cond Res. 2005 Feb;19(1):231-240.

22. Herrington $\mathrm{L}$. Effect of different neurodynamic mobilization techniques on knee extension range of motion in the slump position. J Man Manip Ther. 2006;14(2):101-107.

23. Millesi H, Zöch G, Reihsner R. Mechanical properties of peripheral nerves. Clin Orthop Relat Res. 1995 May;(314):76-83.

24. Rade M, Könönen M, Vanninen R, Marttila J, Shacklock M, Kankaanpää M, et al. 2014 young investigator award winner: In vivo magnetic resonance imaging measurement of spinal cord displacement in the thoracolumbar region of asymptomatic subjects: part 2: comparison between unilateral and bilateral straight leg raise tests. Spine. 2014 Jul 15;39(16):12941300.

25. Jacobson JA, van Holsbeeck MT. Musculoskeletal Ultrasonography. Orthop Clin North Am. 1998;29(1):135-167.

26. Boyd BS, Gray AT, Dilley A, Wanek L, Topp KS. The pattern of tibial nerve excursion with active ankle dorsiflexion is different in older people with diabetes mellitus. Clin Biomech. 2012 Nov;27(9):967-971.

27. Cleland JA, Childs JD, Palmer JA, Eberhart S. Slump stretching in the management of non-radicular low back pain: a pilot clinical trial. Man Ther. 2006 Nov; 11(4):279-286.

28. Nagrale AV, Patil SP, Gandhi RA, Learman K. Effect of slump stretching versus lumbar mobilization with exercise in subjects with non-radicular low back pain: a randomized clinical trial. $J$ Man Manip Ther. 2012;20(1):35-42. 